\title{
Laparoscopic Sleeve Gastrectomy versus Lifestyle Modification in Class I Obesity in Pakistani Population: A Prospective Cohort Study
}

Amina Amin ${ }^{1}$, Ghulam Siddiq ${ }^{1}$, Muhammad Ijlal Haider ${ }^{1}$, Usama Khalid Choudry ${ }^{1}$, Izza Nazir ${ }^{1}$

1. General Surgery, Shifa International Hospital, Islamabad, PAK

$\square$ Corresponding author: Ghulam Siddiq, ghulam_siddiq@yahoo.com

Disclosures can be found in Additional Information at the end of the article

\section{Abstract}

\section{Introduction}

The American Society of Metabolic and Bariatric Surgery has stated that bariatric surgery is indicated in Class I obesity patients with one or more comorbidities. However, other weight loss options, such as diet plus exercise, are available to patients with a body mass index (BMI) ranging from 30 to $35 \mathrm{~kg} / \mathrm{m}^{2}$. This study aimed to prospectively compare the results of Class I obesity patients undergoing laparoscopic sleeve gastrectomy (LSG) or using a weight control program (WCP).

\section{Methods}

A prospective analysis was conducted of patients with Class I obesity and comorbid diabetes and hypertension, with follow-ups at 6, 12, and 18 months. Subjects were divided into two groups: the LSG group of patients who had undergone LSG, and the WCP group who adhered to a WCP. The percentage of excess BMI loss (\%EBMIL) and comorbidity remission (diabetes mellitus and hypertension) were tracked with measurements of hemoglobin A1C (HBA1C) levels and systolic blood pressure. Self-esteem was also tracked using the Rosenberg SelfEsteem Scale (SES) at 0 and 18 months. The overall patient satisfaction score was calculated using a visual analogue scale.

\section{Results}

Of the 150 patients enrolled in the study, 106 were included in the LSG group, and 103 were included in the WCP group. The reduction in HBA1C was more pronounced in the LSG group, and the differences between the two were statistically significant after 6,12 , and 18 months (LSG 5.6 \pm 0.47 vs. WCP $6.5 \pm 0.64$, CI 1.04-0.73, P < 0.05). At 12 and 18 months, there were statistically significant reductions in systolic blood pressure after LSG (LSG 134.2 \pm 7.16 vs. WCP $145.63 \pm 5.94$, CI 13.2-9.6, P < 0.05). Self-esteem levels measured by the Rosenberg SES increased for all participants, while patient satisfaction score was higher in the LSG group than that in the WCP group $(\mathrm{P}<0.05)$. The \%EBMIL at 6 months in the LSG group was $35.48 \%$, compared to the WCP group at only $7.23 \%$. At 12 months, the \%EBMIL had increased twofold in the LSG group, at $68.19 \%$, compared to $14.53 \%$ in the WCP group. At the final 18-month followup, the \%EBMIL in the LSG group was $99.60 \%$ but was only $25.70 \%$ in the WCP group $(\mathrm{P}<0.05)$.

\section{Conclusion}

\section{How to cite this article}

Amin A, Siddiq G, Haider M, et al. (June 28, 2019) Laparoscopic Sleeve Gastrectomy versus Lifestyle Modification in Class I Obesity in Pakistani Population: A Prospective Cohort Study . Cureus 11(6): e5031. DOI 10.7759/cureus.5031 
Our study elucidates a clear superiority of LSG over any structured WCP with regard to weight reduction, improvement in glycemic control, and reduction in blood pressure in Class I obesity patients. Additionally, patients having LSG reported markedly improved self-esteem and satisfaction when compared with those who undertook a WCP.

Categories: Endocrinology/Diabetes/Metabolism, General Surgery, Public Health Keywords: laparoscopic sleeve gastrectomy, weight loss program, diabetes mellitus, hypertension

\section{Introduction}

Pakistan is ranked as the ninth most obese nation in the world, leading to a public health concern. Obesity is further characterized into three classes based on body mass index (BMI), where Class I obesity encompasses those with a BMI in the range of 30 through $34.9 \mathrm{~kg} / \mathrm{m}^{2}$. According to the World Health Organization (WHO), in 2016, more than 1.9 billion adults aged 18 years and older were overweight. Of these, over 650 million adults were obese. In the United States, the number of obese adults increased two-fold between 1980 and 2010 (from 16\% to $36.1 \%$ ) [1]. Obesity predisposes individuals to the risk of metabolic conditions such as diabetes mellitus, hypertension, and cardiovascular disease at a younger age [2]. The goal in treating obesity is to improve quality of life and to increase life expectancy by countering the lifeshortening effects of obesity and its associated comorbidities [3]. There are various methods of combatting obesity, ranging from lifestyle modifications, such as dietary modifications and physical activity, to various metabolic surgeries [4]. Bariatric surgery has evolved in recent decades, and today, new procedures such as sleeve gastrectomy and Roux-en-Y gastric bypass are more commonly utilized than older methods such as duodenal switch, biliopancreatic diversion and gastric band. However, the effects of bariatric surgical procedures on weight loss, hormonal function, and metabolic parameters are still being researched [5]. In this study, we compared the outcomes at one year of patients who underwent laparoscopic sleeve gastrectomy (LSG) and patients who chose lifestyle modification as a modality for weight loss and issues related to obesity.

\section{Materials And Methods}

A prospective cohort study was completed with patients who had a BMI between 30 and $35 \mathrm{~kg} / \mathrm{m}^{2}$. Written and informed consent was received from all participants. The subjects were divided into two groups: the LSG group, which included Class I obesity patients undergoing LSG, and the weight control program (WCP) group, which included Class I obesity patients who undertook a WCP for a minimum duration of 18 months without any gaps. The sample size was calculated as 150 subjects per group. All subjects had follow-ups at 6, 12, and 18 months, and patients who did not complete the follow-ups were excluded from the study. The WCP included dietary modification and daily exercise, recommended and overseen by a nutritionist, where the initial recommendations were a diet of 1300 to $1600 \mathrm{kcal} /$ day and exercise for at least $45 \min 5$ days per week. Daily caloric intake and exercise regimens were accordingly graduated with weight loss results at follow-ups. The main goal of the WCP was to instill healthier behaviors in individuals with Class I obesity.

The primary comparative measure of the study's outcome was the percentage of excess BMI loss (\%EBMIL), and the secondary comparisons of the outcome included measurements of hemoglobinA1C (HBA1c) levels, systolic blood pressure, Rosenberg Self-Esteem Scale (SES) scores, and patient satisfaction ratings. \%EBMIL was calculated as (initial BMI-current $\mathrm{BMI} /($ initial BMI-25)*100. Ideal weight was determined to be a BMI of $25 \mathrm{~kg} / \mathrm{m} 2$. Patients were advised to stop taking antihypertensive medications (if any) 7 days before follow-ups, and HBA1c levels were collected one day before follow-ups. Self-esteem was measured using the Rosenberg SES, which is a standard scale for quantifying self-esteem, and patient satisfaction 


\section{Cureus}

score was calculated using a visual analogue score. Data were expressed as mean \pm standard deviation (SD), and effect modifiers were controlled through stratification. An independent sample T-test was applied for comparison between the groups, and the significance level was determined to be $<0.05$. Analysis was completed using SPSS software, Version 22 (IBM Inc., Armonk, NY, USA).

\section{Results}

Out of 300 patients, 91 were excluded due to loss at follow-up. Of the remaining 209 patients, 106 were included in the LSG group, and 103 were included in the WCP group. The study included $69.9 \%(n=146)$ females, while $36 \%(n=63)$ were males. The mean preoperative levels were as follows: HBA1c was $7.96 \pm 0.82 \%$ in the LSG group and $7.57 \pm 0.65 \%$ in the WCP group; systolic blood pressure was $165 \pm 10.4 \mathrm{mmHg}$ in the LSG group and $162 \pm 11.32 \mathrm{mmHg}$ in the WCP group; BMI was $33.40 \pm 1.03 \mathrm{~kg} / \mathrm{m} 2$ in the LSG group and $32.57 \pm 1.15 \mathrm{~kg} / \mathrm{m}^{2}$ in the WCP group; and the Rosenberg SES was $15.53 \pm 3.39$ in the LSG group and $14.77 \pm 2.64$ in the WCP group. At 6-month follow-up, mean BMI was $30.47 \pm 1.01 \mathrm{~kg} / \mathrm{m}^{2}$ in the LSG group, while in the WCP group, it was $32.04 \pm 1.01 \mathrm{~kg} / \mathrm{m}^{2}$ (CI 1.85-1.29) (P > 0.05). A statistically significant difference was also found at 12 months and 18 months with LSG $27.67 \pm 1.04$ versus WCP $31.47 \pm 0.92$, CI 4.11-3.47, P < 0.05 and LSG $25.03 \pm 1.47$ versus WCP $30.62 \pm 1.02$, CI 5.94-5.24, $\mathrm{P}<0.05$, respectively. There was a significant reduction in mean HBA1c levels in both groups; however, the reduction was more pronounced in the LSG group, and the difference between the two was statistically significant after 6,12 , and 18 months, at LSG $7.26 \pm 0.54$ versus WCP $7.10 \pm 0.59$, CI 0.04-0.31, P = 0.04; LSG $6.45 \pm 0.40$ versus WCP $6.92 \pm 0.61$, CI 0.61-0.33, P $<0.05$; and LSG $5.6 \pm 0.47$ versus WCP $6.5 \pm 0.64$, CI 1.04-0.73, P < 0.05, respectively (figure 1).

Comparison of mean HBA1C levels in both study groups

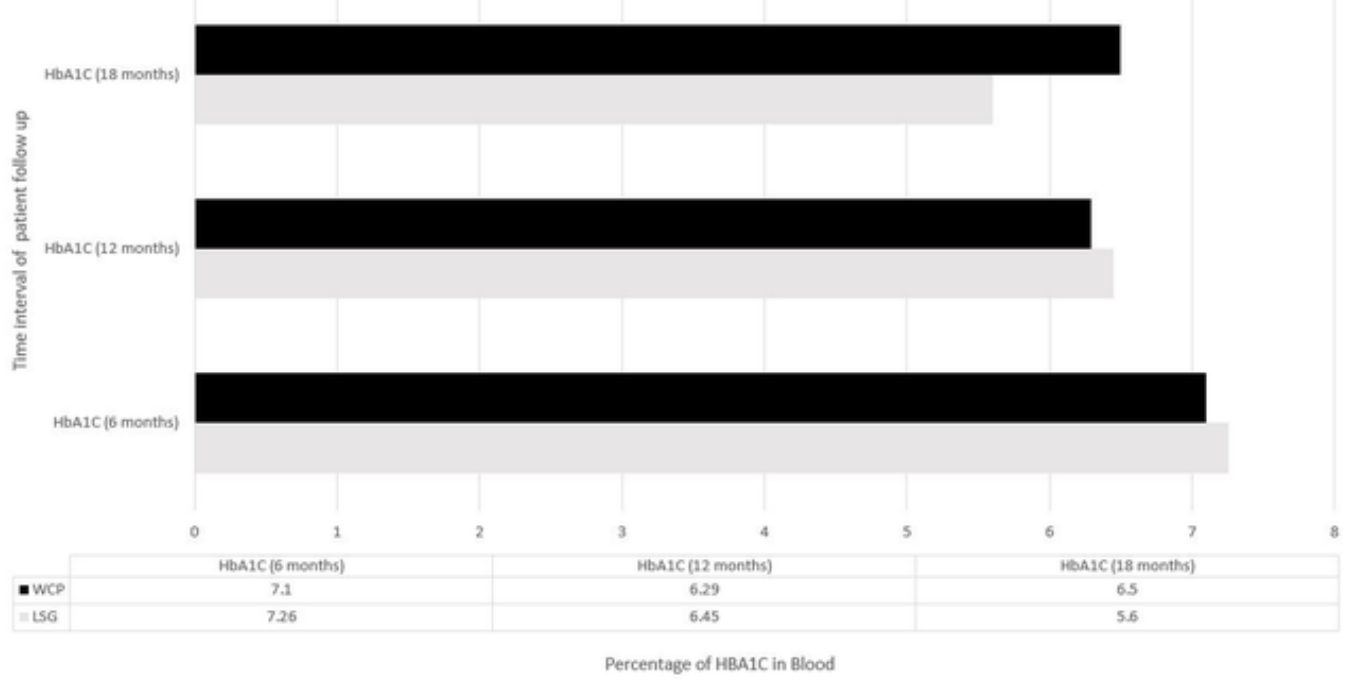

\section{FIGURE 1: Comparison of mean HBA1C in both study groups}

HBA1C : Hemoglobin A1C; WCP : Weight Control Program; LSG : Laparoscopic Sleeve Gastrectomy

There was no statistically significant difference between the groups for blood pressure at 6 months (LSG 161.41 \pm 8.69 vs. WCP 161.1 \pm 8.39 , CI 2.0-2.5, P < 0.05), whereas at 12 and 18 months, there were statistically significant variations in blood pressure, with measurements of LSG 145.7 \pm 7.6 versus WCP 154.1 \pm 6.79 , CI 10.3-6.1, P < 0.05 and LSG 134.2 \pm 7.16 versus 


\section{Cureus}

WCP $145.63 \pm 5.94$, CI 13.2-9.6, P $<0.05$, respectively (figure 2 ).

Comparison of mean systolic blood pressure in both groups

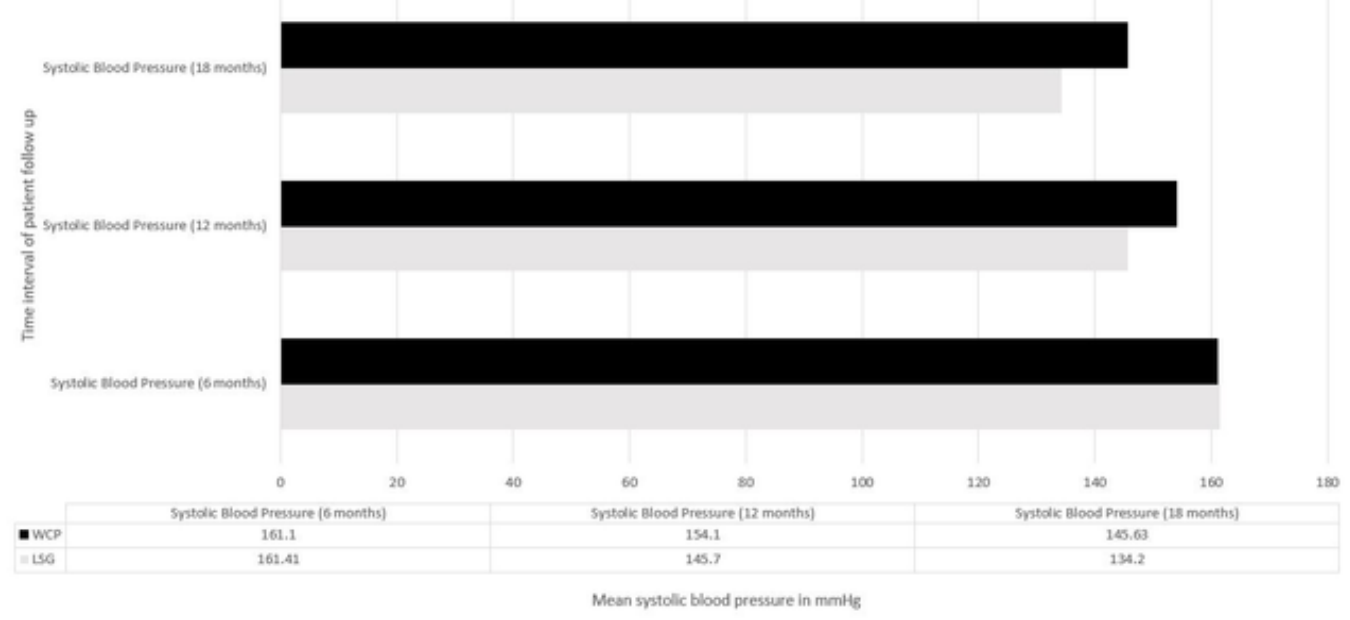

FIGURE 2: Comparison of mean systolic blood pressure in both groups

WCP :Weight Control Program; LSG :Laparoscopic Sleeve Gastrectomy; mmHg: milimetre of mercury

The Rosenberg SES levels increased in the entire study population. However, there was a considerably greater increase in Rosenberg scores at 18 months in the LSG group as compared to the WCP group (LSG $33.04 \pm 3.44$ vs. WCP $22.71 \pm 1.81$, CI 9.56-11.07, P $<0.05$ ). Patient satisfaction scores were also better in the LSG group as compared to the WCP group (LSG $8.2 \pm 0.84$ vs. WCP $6.44 \pm 0.91$, CI 1.55-2.05, P < 0.05). \%EBMIL at 6 months in the LSG group was $35.48 \%$, compared to the WCP group at only $7.23 \%$. At 12 months, \%EBMIL had increased two-fold in the LSG group to $68.19 \%$, while it was at $14.53 \%$ in the WCP group. At the final 18 month follow-up, \%EBMIL in the LSG group was $99.60 \%$, while it was only $25.70 \%$ in the WCP group $(\mathrm{P}<0.05)$ (figure 3).

Comparison of \%EBMIL between LSG and WCP group

- IsG = wCP

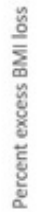

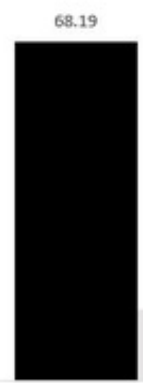

14.53

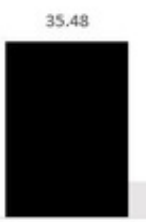

7.23

EBMIL-6

EBMIL-12

Time interval of patient follow up

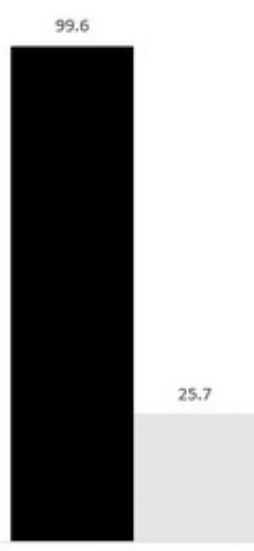

EBMIL-18 


\section{Cureus}

FIGURE 3: Comparison of \%EBMIL between WCP and LSG group

\%EBMIL : Percent Excess Body Mass Index Loss; WCP: Weight Control Program; LSG: Laparoscopic Sleeve Gastrectomy

The statistical results of the study have been summarized in Table 1 .

\begin{tabular}{|c|c|c|c|c|}
\hline Mean Measurements & LSG Group & WCP Group & $\mathrm{Cl}$ & P-Value \\
\hline Age & 41.7 years & 38.8 years & $41.72-39.41$ & 0.01 \\
\hline Gender & Male: $n=34$ Female: $n=72$ & Male: $n=33$ Female: $n=70$ & $1.6-1.7$ & 0.01 \\
\hline Preoperative HBA1C & $7.96 \%$ & $7.87 \%$ & $0.86-0.60$ & 0.627 \\
\hline Preoperative Systolic BP & $171.3 \mathrm{mmHg}$ & $169.8 \mathrm{mmHg}$ & $0.75-1.43$ & 0.116 \\
\hline Initial BMI & $33.3 \mathrm{~kg} / \mathrm{m}^{2}$ & $32.5 \mathrm{~kg} / \mathrm{m}^{2}$ & $0.49-1.10$ & 0.05 \\
\hline BMI, 6 Months & $30.4 \mathrm{~kg} / \mathrm{m}^{2}$ & $32.0 \mathrm{~kg} / \mathrm{m}^{2}$ & $1.85-1.29$ & 0.05 \\
\hline BMI, 12 Months & $27.6 \mathrm{~kg} / \mathrm{m}^{2}$ & $31.4 \mathrm{~kg} / \mathrm{m}^{2}$ & $4.12-3.47$ & 0.05 \\
\hline BMI, 18 Months & $25.0 \mathrm{~kg} / \mathrm{m}^{2}$ & $30.6 \mathrm{~kg} / \mathrm{m}^{2}$ & $5.94-5.24$ & 0.05 \\
\hline HBA1C, 6 Months & $7.26 \%$ & $7.23 \%$ & $0.13-0.18$ & 0.72 \\
\hline HBA1C, 12 Months & $6.45 \%$ & $6.92 \%$ & $0.61-0.33$ & 0.05 \\
\hline HBA1C, 18 Months & $5.68 \%$ & $6.57 \%$ & $1.04-0.73$ & 0.05 \\
\hline Systolic BP, 6 Months & $161.4 \mathrm{mmHg}$ & $161.1 \mathrm{mmHg}$ & $2.08-2.58$ & 0.83 \\
\hline Systolic BP, 12 Months & $145.7 \mathrm{mmHg}$ & $154.1 \mathrm{mmHg}$ & $10.30-6.44$ & 0.05 \\
\hline Systolic BP, 18 Months & $134.2 \mathrm{mmHg}$ & $145.6 \mathrm{mmHg}$ & $13.2-9.6$ & 0.05 \\
\hline Self-Esteem Score, Day 0 & 14.9 & 14.7 & $0.69-0.99$ & 0.73 \\
\hline Self-Esteem Score, 18 Months & 33.0 & 22.7 & $9.56-11.00$ & 0.05 \\
\hline Patient Satisfaction Score & 8.26 & 6.44 & $1.57-2.05$ & 0.05 \\
\hline
\end{tabular}

\section{TABLE 1: Mean measurements taken throughout the study period}

BMI: Body Mass Index; BP: Blood Pressure; Cl: Confidence Interval; LSG: Laparoscopic Sleeve Gastrectomy; WCP: Weight Control Program

\section{Discussion}


The Pakistani population has witnessed an increase in the number of individuals suffering from obesity over time [6]. There has also been a rising trend in the use of bariatric surgery as a treatment modality for obesity in developing Asian countries since obesity has become a public health concern. The literature shows that the loss of excess weight has proven benefits in terms of the metabolic profile of patients suffering from obesity. Batsis et al. reported a significant reduction in the number of individuals with metabolic syndrome after bariatric surgery (from $87 \%$ down to 29\%) [7]. There have also been studies proving the beneficial effects of weight loss for diabetes control. In our study population, the LSG group mean HBA1c level was reduced to a non-diabetic level after bariatric surgery. However, the mean HBA1c level in the WCP group was still in the diabetic range. In a meta-analysis of 357 patients, a significant decrease in HBA1c levels (2.59\%) was noted among patients with Class I obesity who underwent bariatric surgery [8]. Another systematic review cited a linear relationship between the reduction of HBA1c levels and various weight loss methods, with an overall reduction of $0.1 \% / \mathrm{kg}$ [9]. The results of these studies show an eminent superiority of bariatric surgery over WCPs with respect to glycemic control. Weight loss has also been reported to act on several neurohormonal mechanisms to control hypertension through, for instance, decreased oxidative stress, decreased insulin resistance, and decreased aldosterone release [10]. With regard to the comparison of hypertension as part of the metabolic profile, in our study, the majority of patients in the LSG group became normotensive by the 18-month follow-up (LSG $134.2 \pm 7.16 \mathrm{mmHg}$ ), while in the WCP group (145.63 $\pm 5.94 \mathrm{mmHg})$, the majority still needed medication to control blood pressure. This is in agreement with a meta-analysis that included 14 studies (3,550 subjects) showing a resolution of hypertension in $62.17 \%$ and improvement in $35.70 \%$ of participants at the 5-year follow-up after sleeve gastrectomy [11]. Similarly, another meta-analysis of 25 studies found a direct link between weight loss and reduction of hypertension. Further, a decrease of $1 \mathrm{~kg}$ of weight has been associated with an approximate $1 \mathrm{mmHg}$ decline in systolic blood pressure [12]. In the Asian population, individuals with Class I obesity who have undergone LSG have shown a greater \%EBMIL than other treatment modalities [13]. In our study, the LSG group had a more rapid change in \%EBMIL at 6,12, and 18 months and reached nearly 100\%, in comparison to the WCP group, where the trend of \%EBMIL was very gradual and only reached $25 \%$ by the end of 18 months. According to a meta-analysis of randomized controlled trials and cohort studies conducted in 2018, there was no highquality evidence for treating obesity with WCPs because the level of benefits using such methods was minimal when contrasted with the weight loss that was needed [14]. However, one study noted that individuals using lifestyle modification to achieve weight loss surprisingly maintained $10 \%$ of excess weight loss throughout the duration of the study period [15]. This result did not relate to the main objective, but it shows that WCPs could help patients in controlling the disease. However, it must be taken into consideration that subjects treated with WCPs still have a BMI of $30 \mathrm{~kg} / \mathrm{m} 2$ or more. A similar study comparing lifestyle intervention with bariatric surgery in Class I obesity patients showed similar results, with a \%EBMIL of $16.20 \%$ and $17.90 \%$ at 1 and 3 years, respectively, in the lifestyle intervention group [15], which compares with a \%EBMIL of $14.53 \%$ and $25.70 \%$ at 12 and 18 months, respectively, in our WCP group. Additionally, \%EBMIL of $67.90 \%$ and $76.90 \%$ at 1 and 3 years, respectively, in their bariatric surgery group is comparable to our study's \%EBMIL of $68.19 \%$ and $99.60 \%$ at 12 and 18 months, respectively, in the LSG group. Obesity has a tremendous social and psychological impact as well. The Rosenberg SES scores were initially $15.53 \pm 3.39$ in our LSG group and $14.77 \pm 2.64$ in our WCP group. At the third follow-up (18 months), there had been a significant rise in self-esteem scores in the LSG group to $33.04 \pm 3.44$. Similarly, a prospective cohort study involving 32 individuals undergoing sleeve gastrectomy reported a significant rise in selfesteem scores $(\mathrm{P}$ value $=0.003$ ) after 1 year [16]. A study of obese women by Dennis et al. also reported improved self-esteem and mood after a structured WCP, in agreement with our study [17]. Furthermore, the overall patient satisfaction in our LSG group was comparable to a large cohort study of 110 sleeve gastrectomy patients (8.2/10 vs. 8/10, respectively) [18].

The limitations of our study include smaller sample size and a short follow-up duration. 
However, because this is the first study from Pakistan on this topic, our results make a vital contribution to the literature. Additionally, we plan to do an extended follow-up study with a larger cohort of patients.

\section{Conclusions}

Our study elucidates a clear superiority of LSG over a structured WCP with regard to weight reduction, improvement in glycemic control, and reduction in blood pressure among Class I obesity patients. Additionally, this study showed that patients reported markedly improved self-esteem and satisfaction after bariatric surgery as compared to lower levels of improvement with a WCP, pointing to bariatric surgery as a preferred mode of treatment for Class I obesity.

\section{Additional Information}

\section{Disclosures}

Human subjects: Consent was obtained by all participants in this study. Institutional review board and Ethics committee,Shifa International Hospital. issued approval N/A. We would like to inform you that study "Laparoscopic Sleeve Gastrectomy versus Lifestyle Modification in Class I Obesity in Pakistani Population: A Prospective Cohort Study" has been approved.The IRB/EC is in accordance to ICH and GCP guidelines.Any changes in the protocol should be notified to the committee for prior approval.All the informed consents should be retained for future reference (if applicable).A proper report should be submitted quarterly and final report after the completion of the study to the IRB and Ethics Committee. Your's Sincerely, Dr Ejaz A Khan. Chairman, IRB \& EC . Animal subjects: All authors have confirmed that this study did not involve animal subjects or tissue. Conflicts of interest: In compliance with the ICMJE uniform disclosure form, all authors declare the following: Payment/services info: All authors have declared that no financial support was received from any organization for the submitted work. Financial relationships: All authors have declared that they have no financial relationships at present or within the previous three years with any organizations that might have an interest in the submitted work. Other relationships: All authors have declared that there are no other relationships or activities that could appear to have influenced the submitted work.

\section{References}

1. Cefalu WT, Bray GA, Home PD, et al.: Advances in the science, treatment, and prevention of the disease of obesity: reflections from a Diabetes Care editors' expert forum. Diabetes Care. 2015, 38:1567-1582. 10.2337/dc15-1081

2. Inge TH, Krebs NF, Garcia VF, et al.: Bariatric surgery for severely overweight adolescents: concerns and recommendations. Pediatrics. 2004, 114:217-223. 10.1542/peds.114.1.217

3. Dietrich A, Aberle J, Wirth A, Müller-Stich B, Schütz T, Tigges H: Obesity surgery and the treatment of metabolic diseases. Dtsch Arztebl Int. 2018, 115:705-711.

10.3238/arztebl.2018.0705

4. Tham KW, Lee PC, Lim CH: Weight management in obstructive sleep apnea: medical and surgical options. Sleep Med Clin. 2019, 14:143-153. 10.1016/j.jsmc.2018.10.002

5. Buchwald H: The evolution of metabolic/bariatric surgery . Obes Surg. 2014, 24:1126-1135. 10.1007/s11695-014-1354-3

6. Shah AA, Shariff AH: Obesity and the need for bariatric surgery in Pakistan. Asian J Endosc Surg. 2013, 6:257-265. 10.1111/ases.12048

7. Batsis JA, Romero-Corral A, Collazo-Clavell ML, Sarr MG, Somers VK, Lopez-Jimenez F: Effect of bariatric surgery on the metabolic syndrome: a population-based, long-term controlled study. Mayo Clin Proc. 2008, 83:897-906. 10.4065/83.8.897

8. Li Q, Chen L, Yang Z, et al.: Metabolic effects of bariatric surgery in type 2 diabetic patients with body mass index < 35 kg/m2. Diabetes Obes Metab. 2012, 14:262-270. 10.1111/j.14631326.2011.01524.x

9. Gummesson A, Nyman E, Knutsson M, Karpefors M: Effect of weight reduction on glycated 
haemoglobin in weight loss trials in patients with type 2 diabetes. Diabetes Obes Metab. 2017, 19:1295-1305. 10.1111/dom.12971

10. Cohen JB: Hypertension in obesity and the impact of weight loss . Current Cardiol Rep. 2017, 1:98. 10.1007/s11886-017-0912-4

11. Graham C, Switzer N, Reso A, et al.: Sleeve gastrectomy and hypertension: a systematic review of long-term outcomes. Surg Endosc. 2018, 1:1-7. 10.1007/s00464-018-6566-5

12. Sabaka P, Dukat A, Gajdosik J, Bendzala M, Caprnda M, Simko F: The effects of body weight loss and gain on arterial hypertension control: an observational prospective study. Eur J Med Res. 2017, 22:43. 10.1186/s40001-017-0286-5

13. Ismail M, Nagaraj D, Rajagopal M, Ansari H, Nair M, Hegde A, Rekha PD: Seven-year outcomes of laparoscopic sleeve gastrectomy in Indian patients with different classes of obesity. Obes Surg. 2019, 29:191-196. 10.1007/s11695-018-3506-3

14. Solmi M, Köhler CA, Stubbs B, et al.: Environmental risk factors and nonpharmacological and nonsurgical interventions for obesity: an umbrella review of meta-analyses of cohort studies and randomized controlled trials. Eur J Clin Invest. 2018, 48:12982. 10.1111/eci.12982

15. Vitiello A, Angrisani L, Santonicola A, Iovino P, Pilone V, Forestieri P: Bariatric surgery versus lifestyle intervention in class I obesity: 7-10-year results of a retrospective study. World J Surg. 2019, 43:758-762. 10.1007/s00268-018-4847-8

16. Aldaqal SM, Sehlo MG: Self-esteem and quality of life in adolescents with extreme obesity in Saudi Arabia: the effect of weight loss after laparoscopic sleeve gastrectomy. Gen Hosp Psychiatry. 2013, 35:259-264. 10.1016/j.genhosppsych.2012.12.011

17. Dennis KE, Goldberg AP: Weight control self-efficacy types and transitions affect weight-loss outcomes in obese women. Addict Behav. 1996, 21:103-116. 10.1016/0306-4603(95)00042-9

18. Arman GA, Himpens J, Dhaenens J, Ballet T, Vilallonga R, Leman G: Long-term (11+ years) outcomes in weight, patient satisfaction, comorbidities, and gastroesophageal reflux treatment after laparoscopic sleeve gastrectomy. Surg Obes Related Diseases. 2016, 12:17781786. 10.1016/j.soard.2016.01.013 